\title{
Tres Saberes en la Formación Profesional por Competencias: Integración de Teorías Subjetivas, Profesionales y Científicas
}

\author{
David J. Cuadra-Martínez ${ }^{1}$ Pablo J. Castro ${ }^{3}$ y María T. Juliá ${ }^{2}$ \\ 1 Universidad de Atacama, Facultad de Humanidades y Educación, Departamento de Psicología, Copayapu \\ 485, Copiapó-Chile. (e-mail: david.cuadra@uda.cl) \\ 2 Universidad de La Serena, Facultad de Humanidades, Departamento de Psicología, Matta 147, Coquimbo- \\ Chile. (e-mail: pablocastro@userena.cl; zmtjulia@gmail.com) \\ ${ }^{3}$ Universidad Católica del Maule, San Miguel 3605, Talca-Chile
}

Recibido Dic. 26, 2017; Aceptado Mar. 14, 2018; Versión final Abr. 20, 2018, Publicado Oct. 2018

\begin{abstract}
Resumen
El objetivo de este trabajo es analizar el rol que las teorías subjetivas, profesionales y científicas tienen en la formación profesional y presentar una propuesta para lograr que se integren entre ellas, en el contexto de un enfoque de formación basado en competencias. A partir de un análisis de estudios empíricos y teóricos sobre la formación profesional, se argumenta que los profesionales ejercen su acción profesional, orientados por tres tipos de saberes: teorías científicas, profesionales y subjetivas. Sin embargo, en la investigación y formación profesional, a estas últimas teorías se las ha abordado escasamente. De esto se concluye sobre la necesidad de incluir e integrar sistemáticamente en la investigación y formación profesional a estos tres saberes. De esta forma, se contribuye a que los profesionales aborden de manera más efectiva los complejos problemas sociales y ambientales que nuestra sociedad enfrenta hoy en día.
\end{abstract}

\section{Three Types of Knowledge in Competency Based Professional Education: The Integration of Subjective, Professional, and Scientific Theories}

\begin{abstract}
The aim of this study was to analyse the role of subjective, professional, and scientific theories in professional training, and to develop a proposal that integrates them in the context of a competency-based approach. Based on analyses of empirical and theoretical studies on professional training, it is argued that professionals shaped their professional performances from three types of knowledge: scientific, professional and subjective theories. These last two types of knowledge have scarcely been addressed. From this, it is concluded that it is necessary to integrate them into a systematic approach that involves research and academic training. In this way, we can contribute that professionals deal more effectively with the complex social and environmental problems that our society nowadays faces.
\end{abstract}

Keywords: university education; competency-based education; scientific theories; professional theories; subjective theories 


\section{INTRODUCCIÓN}

Han pasado más de 20 años desde que se ha estado escribiendo sobre la formación basada en competencias (Tobón, 2001). Sin embargo y a pesar de su extendido uso en la formación universitaria de nuestros países, aún no se aprecia un cambio evidente en el ejercicio de los profesionales que han recibido este tipo de formación. La relación entre la formación universitaria y el ejercicio profesional es un problema de larga data que la teoría de las profesiones no ha considerado con la suficiente preocupación (Nolin, 2008). De la misma manera, el conocimiento académico que se genera y se enseña en las universidades no ha valorado el conocimiento profesional (el de los profesionales en ejercicio) con un estatus que permita su incorporación y diálogo con la formación universitaria (Donoso y Corvalán, 2012; Gergen, 1996).

Schomburg y Teichler ( 2006) planteaban que los estudios universitarios en europeos tenían poco uso en el empleo y las expectativas desde el puesto de trabajo no son satisfechas por las competencias de egreso. Otro ejemplo es un estudio realizado en siete universidades chilenas para validar las competencias de egreso del profesional psicólogo, en donde al entrevistar a estudiantes, docentes, tutores, empleadores y egresados, se muestra una brecha entre el ámbito académico y el de la práctica de la profesión (Red Psicología CUECH Proyecto MECESUP ULS 0601, en Juliá 2013). En este contexto, las universidades han transitado desde declarar un modelo de enseñanza centrado en la formación de capacidades, a la adquisición de cualificaciones individuales, derivando finalmente en la predominancia hoy de un enfoque de formación profesional basado en competencias (Bustamante et al., 2017).

Este último enfoque exige un aprendizaje complejo en el estudiante, porque se busca el desarrollo integral del mismo, que para Hill y Houghton (2001) involucra promover en los futuros profesionales una autoconciencia metacognitiva y un aprendizaje experiencial que se transmita más allá del aula. Este aprendizaje es "complejo", entre otros aspectos, porque requiere de la integración en el aprendiz de saberes científicos, cotidianos y profesionales, en un mismo escenario de formación y posterior ejercicio profesional. Pérez y Pérez (2013) plantean que la competencia es una forma de saber que nace de dentro, de la experiencia vivida, pensada, reflexionada y contrastada: "El carácter holístico, reflexivo y contextualizado constituye el eje esencial de las competencias" (p. 70). De igual forma, a la docencia universitaria se le exige un cambio de foco que, con relativa independencia de si se enfatizan más o menos los contenidos, consiste en proponerse como objetivo formar capacidades de acción y de reflexión a partir de los contenidos enseñados. En este sentido, se propone que la formación profesional incorpore toda la experiencia y los conocimientos multidisciplinares y multidimensionales que se poseen. Esto supone que la competencia se desarrolla a partir de una serie de saberes por parte del estudiante, que se requieren sistematizar, concientizar, racionalizar y reconstruir para dar solución a los problemas que enfrentan (Frade, 2009).

Esta orientación curricular debiera considerar las características y la diversidad del estudiantado para, de este modo, "conjugar las metas de calidad y equidad educativa" (Sanz de Acedo, 2010, p. 18). Sin embargo, la implementación del enfoque de formación en base a competencia no ha estado exenta de dificultades (Pérez y Pérez, 2013). Una de estas se relaciona con la formación del profesor y la concepción predominante de enseñanza que implica más la transmisión de contenidos teórico técnicos al estudiante (Juliá, 2015), dejando de lado el desarrollo de otras competencias que son fundamentales para el desempeño idóneo (Tobón, 2008).

El saber científico-académico es el tipo de conocimiento que fundamentalmente se ha transmitido y sigue enseñando en las instituciones educativas (Aguerrondo, 2009). Es entendido aquí como aquel que surge desde el método científico y que permite comprender y explicar los fenómenos sociales y naturales, para reconocer las leyes de la realidad, mediante teorías. Desde la teoría de las profesiones, la perspectiva funcionalista ha puesto un fuerte énfasis en la distinción de una profesión, en base a su conocimiento científico y técnico (Nolin, 2008). Pero el saber subjetivo también es constituyente de la dimensión humana y de los procesos formativos. Las personas funcionan como "científicos intuitivos" (Rodrigo, 1997), elaborando explicaciones o teorías subjetivas (TS) que les permiten representarse a sí mismo y el mundo, además de organizar y orientar la acción (Catalán, 2016; Flick, 2014; Groeben y Scheele, 2000). Las TS inciden de manera significativa en la formación inicial, regulando en algún nivel el aprendizaje y la disposición hacia el mismo (Šteh et al., 2014). También permiten explicar y predecir fenómenos propios de la profesión, además de justificar y orientar la acción profesional (Kindermann y Riegel, 2016). Por otra parte, la necesidad de lograr una mayor integración entre el saber científico-académico y la práctica profesional (Tobón et al., 2010), exige considerar el conocimiento que los profesionales elaboran a partir de su ejercicio profesional, mediante procesos reflexivos en y durante la acción (Schön, 1984). Este saber profesional situado, a veces subjetivo y otras veces consensuado u objetivado socialmente, puede ser factible de interpretar como verdaderos modelos de conocimientos explicativos o teorías profesionales (TP) que guían la acción, hipótesis que el profesional utiliza para resolver problemas profesionales que demandan su atención. 
Desde lo anterior, parece oportuno proponer la tesis de que los profesionales ejercen su acción profesional, orientados por teorías científicas (TC), profesionales (TP) y subjetivas (TS), pero en el proceso formativo y la investigación, a estas últimas se las ha abordado escasamente (Campillo et al., 2012) y principalmente desde la sociología (Nolin, 2008). Se hace necesario incluir sistemáticamente en la investigación y formación inicial estas dimensiones psicológicas de conocimientos si se quiere lograr un cambio en la formación profesional, sobre todo desde un modelo de formación por competencias. El objetivo de este ensayo es analizar el rol que estos tipos de saberes tienen en la formación profesional y proponer algunas ideas acerca de cómo incorporarlos en un enfoque por competencias.

\section{LA FORMACIÓN PROFESIONAL BAJO EL ENFOQUE POR COMPETENCIAS}

Competencia se ha definido según las distintas tradiciones o paradigmas (conductista, funcionalista, constructivista y sistémico-complejo) que han abordado el concepto (Tobón, 2008). En términos generales se tiende a asumir posiciones más psicológicas, destacando el término capacidad; o posiciones más contextuales, poniendo como foco el desempeño situado y la intersubjetividad (Juliá, 2015). Así, encontramos conceptos como el de actuación, capacidad, procesos complejos para un desempeño con idoneidad en determinados contextos.

Las competencias son combinaciones dinámicas de recursos personales, complejos sistemas de comprensión y acción que incluyen «saber pensar», "saber decir», «saber hacer» y «querer pensar, decir y hacer». Se ponen en juego para comprender la complejidad de las situaciones en las que se pretende actuar y para diseñar, planificar, desarrollar y evaluar los modos concretos de actuación. La acción en situaciones concretas y complejas de la vida profesional conlleva a un "saber de la experiencia", que produce una mayor comprensión de la experiencia vivida, pensada, reflexionada y contrastada (Pérez y Pérez, 2013). Este enfoque propone planificar la enseñanza en base al desarrollo de competencias en el estudiantado, unidad organizadora de los perfiles profesionales, mallas curriculares y programas de estudio.

Se constituye más como un enfoque o propuesta para formar al profesional actual, porque no representa en sus planteamientos a todo el proceso educativo y apunta al logro de un desempeño complejo e idóneo, que incluye saber ser, saber hacer, saber conocer y saber convivir, aportando al progreso personal, social y económico (Tobón, 2008, Juliá 2013). En general, busca lograr una estrecha relación entre lo que el estudiante aprende en educación superior y los desafíos que imponen los contextos sociales y laborales (Lago y Ospina, 2015). Se buscan saberes de segundo orden de tipo metacognitivos que se evidencien en la transferencia del conocimiento, actitudes, destrezas y habilidades, a situaciones nuevas (Juliá, 2015). Desde este marco, se requieren nuevas concepciones de aprendizaje. "La formación de las competencias... no solo debe considerar el diseño instrumental, metodológico o estratégico, sino también el análisis crítico y reflexivo del campo de acción y de trabajo" (Juliá, 2015, p. 48). También se necesitan nuevos roles educativos: (a) la formación profesional se piensa en base a estándares que permiten evaluar el desempeño en el puesto de trabajo (Mukhongo, 2015); (b) el aprendizaje es un proceso en donde el estudiante asume un rol protagónico y activo (Bustamante et al., 2017; Juliá, 2015); y (c) el profesor tiene la meta de formar profesionales reflexivos, creativos, éticos, comprometidos, más eficientes, en definitiva, integrales.

Respecto de la enseñanza, ésta se concibe como un proceso flexible y adecuado al estudiante, que reconoce la heterogeneidad y su cultura de formación (Juliá, 2015). El énfasis está puesto en la enseñanza de procesos, estrategias y habilidades de pensamiento utilizando el conocimiento disciplinario y cultural como un medio, centrando la enseñanza en el aprendizaje. El resultado de este proceso es el desarrollo de competencias para dominar una tarea, una mayor confianza de parte de los estudiantes respecto de su desempeño (Mukhongo, 2015), incluso en situaciones de incertidumbre, que requieren creatividad y transformación de la información en un saber (Juliá, 2015).

\section{LAS TEORÍAS CIENTÍFICAS EN LA FORMACIÓN PROFESIONAL}

El conocimiento profesional ha sido definido como una integración compleja de conocimientos y habilidades necesarios para desempeñar una profesión (Tamir, 1991). Se caracteriza por ser práctico, dado que guía la acción, además de situado y contextualizado, porque emerge desde ésta y la cultura en que es utilizado. También es un conocimiento complejo, por su dinamismo e integración de diversos saberes que surgen desde variadas fuentes de conocimiento, siendo una de ellos las TC (Sosa y Ribeiro, 2015).

A pesar del consenso respecto a la complejidad del saber profesional, el enfoque de formación basado en competencias, advierte sobre la excesiva abstracción de las TC y su baja relación con la práctica en los procesos formativos. Por ejemplo, Pérez y Pérez (2013) afirman que para el caso de la formación docente, el conocimiento académico, abstracto, fragmentado y descontextualizado, presente en la mayoría de los 
programas de formación, no induce aplicaciones concretas ni provoca, por tanto, la reconstrucción de las concepciones previas de los estudiantes, su pensamiento práctico.

El mundo científico tampoco ha estado exento de esta crítica: actualmente se viene impulsando la producción de conocimiento científico de una manera diferente a la tradicional, coherente con las demandas de la sociedad del conocimiento y las exigencias del mercado (Gallegos et al., 2014). Se intenta avanzar hacia una ciencia post académica, más preocupada por lograr una mayor coherencia y aplicabilidad de las TC, en donde su producción sea más participativa, además de posnormal, es decir, que parta de la premisa de la complejidad de la realidad socio ambiental, que para Morín (2010) implica el desarrollo de un pensamiento complejo para abordar los fenómenos del medio.

Este intento de adecuación de la forma de desarrollar TC, responde a la baja pertinencia de éstas con los problemas sociales y ambientales y, en la educación superior, a su limitado impacto en la formación profesional (Campillo et al., 2012; Schön, 1984). Así, es necesario considerar que la realidad es un escenario complejo (Morín, 2010) y como consecuencia de esto, un elemento distintivo de la profesión es trabajar en condiciones de riesgo e incertidumbre (Evetts, 2014). Los(as) profesionales se desenvuelven en un ambiente altamente dinámico, complejo y problemático (Schön, 1984), frente al cual se requieren de otros tipos de conocimientos, que funcionan en escalones distintos de abstracción y sistematización y por lo tanto, aplican a distintos problemas y situaciones. "La ciencia provee conocimientos que son parte de aquello que colabora con la práctica pero no la totaliza, es sólo un ingrediente, un elemento que está en juego sin agotar todo lo que está en la práctica" (Follari, 2016, p. 43). Desde esto, el papel de las TC en la formación profesional, incluso en las "profesiones clásicas" (Campillo et al., 2012, p. 8), es el de proveer de algunos modelos explicativos desde los cuales se puede tomar decisiones fundadas para la acción profesional, porque poseen evidencia empírica de los hechos, sin embargo, estas teorías no resuelven gran parte de los problemas que enfrentan los profesionales e incluso, parece ser que los de mayor relevancia escapan a la TC (Schön, 1984).

Por otro lado y siguiendo a Gergen (1996) pareciera ser el momento (hace más de 20 años según el autor) en que los especialistas académico-científicos, se beneficien de los modelos explicativos y de toma de decisiones de quienes desarrollan conocimiento profesional. Este tipo de conocimiento, que puede ser subjetivo o también objetivado socialmente, podría tener un impacto cultural mayor gracias a su contextualización y trabajo diario con las personas y organizaciones. En la misma línea Pérez y Pérez (2013) plantean, para el caso de las competencias de los docentes, por ejemplo, que el pensamiento práctico es especialmente importante porque es aquel que gobierna los modos de intervenir sobre la realidad, transformándola y mejorándola a través de procesos continuos de reflexión y crítica.

\section{TEORÍAS SUBJETIVAS: MODELOS QUE GUÍAN LA FORMACIÓN Y LA ACCIÓN}

"Si las teorías explícitas y declaradas no conectan con las teorías implícitas, con los esquemas, recursos, hábitos y modos intuitivos de percibir, interpretar, anticiparse y reaccionar, se convierten en meros adornos, útiles en todo caso para la retórica o para la superación de exámenes, pero estériles para gobernar la acción en las situaciones complejas, cambiantes, inciertas y urgentes del aula" (Pérez, 2010, p. 46).

Los profesionales guían su acción profesional en base a un saber subjetivo, más cercano a la vida cotidiana y a la experiencia, cuando las TC y el conocimiento técnico por si solos no le permiten otorgar respuesta a los problemas que requieren resolver. Esto, sobre todo cuando estos problemas implican respuestas morales y axiológicas. De esta forma, los profesionales podrían desarrollar “... percepciones y sentimientos conducidos por los implicados en ella, dando lugar a una serie de interacciones y dinámicas dialógicas en donde más que aplicarse conocimiento estándar, técnico y objetivo, se recrean de acuerdo con los modos de pensar de los participantes" (Campillo et al., 2012, p. 10).

Lo anterior ha sido estudiado en profesionales desde el enfoque de las TS (Castro et al., 2015; Flick, 2014). Las TS son hipótesis que las personas elaboran en su vida cotidiana (Flick, 2014), a partir de la primera infancia (Catalán, 2016) y desde la experiencia y la interacción social. Permiten comprender el propio comportamiento y el mundo, guiar y justificar la acción (Catalán, 2016; Flick, 2014; Groeben y Scheele, 2000). Pueden encontrarse implícita o explícitamente y corresponden a aquel conocimiento subjetivo de mayor elaboración y arraigo.

Los estudiantes que se forman profesionalmente presentan obviamente también TS acerca de los fenómenos: explican, por ejemplo, fenómenos socio ambientales intuitivamente, desde un saber que es usado y retroalimentado por sus experiencias en la vida cotidiana (Šteh et al., 2014) y que les es funcional. Como se señaló anteriormente, los profesionales también utilizan TS para abordar problemas laborales: explican intuitivamente, desde un saber cotidiano y mucho más implícito, fenómenos que emergen en 
contexto laboral, guiando su acción en base a éstas (Kindermann y Riegel, 2016). Entonces, una formación profesional en base a competencias, debe considerar esta dimensión subjetiva: (a) porque las TS son parte de la dimensión de significados atribuidos a sí mismo y los fenómenos del medio y por el solo hecho de considerarlas en el proceso de enseñanza y aprendizaje, se logra una participación activa y comprometida del estudiante en su proceso formativo; (b) para que el futuro profesional logre un autoconocimiento de la implicancia que tienen sus TS en la acción profesional; (c) enjuicie su funcionalidad; y (d) las reconstruya o enriquezca cuando sea necesario. Así, en la formación profesional se requiere promover, en base a procesos reflexivos a partir de contenidos y/o prácticas, una integración entre las TS relacionadas con la profesión, las TS idiosincráticas de los estudiantes y las científicas. Se trata de generar un cambio subjetivo, en donde el saber científico y académico cobra sentido desde estos modelos explicativos de los estudiantes, logrando el desarrollo de un pensamiento más complejo (Morín, 2010) para la acción profesional.

\section{LAS TEORÍAS PROFESIONALES COMO FAROS DE LA PRÁCTICA PROFESIONAL}

"En muchos aspectos, los terapeutas, los consejeros y los asesores de organización, los especialistas en educación y similares tienen un impacto mucho mayor en la vida cultural que los académicos. Sus acciones pueden participar en prácticas relacionales de un modo más profundo y directo que los escritos abstrusos de los profesionales... A mi entender, la próxima década puede ser aquella en la que el especialista se beneficie más de habilidades contextualizadas del practicante, y no al revés" (Gergen, 1996, p. 57).

En este trabajo, se entiende a las TP, como aquellos modelos explicativos que se desarrollan, aprenden y enseñan en contextos de práctica profesional, a partir de la interacción y el ejercicio profesional. Son aquellas explicaciones que tienen algún grado de sistematización, que se someten a prueba en la comunidad profesional, utilizan un lenguaje técnico propio de la profesión y se transmiten mediante la socialización profesional (Andreozzi, 2011), pero que no han seguido el método científico para elaborarlas, aunque sí una racionalidad, porque el profesional en la acción le otorga sentido a los problemas que enfrenta, reflexiona sobre su práctica pasada y la actual, elaborando hipótesis y probándolas, las que dan origen a una nueva forma de explicar fenómenos.

Por lo tanto, son modelos explicativos, a veces subjetivos y otras veces consensuados $u$ objetivados socialmente, que surgen en el ejercicio profesional para explicarse y orientarse en éste. No son modelos científicos, porque no han seguido las reglas de generación y difusión del conocimiento científico. Desde esto último, puede juzgarse su validez, confiabilidad o credibilidad, no obstante, son funcionales, pertinentes y comprensibles en el quehacer profesional. En palabras de Andreozzi (2011, p. 107), "Los entornos reales de actuación profesional emergen - así - como espacios de producción y circulación de saberes que definen cómo son y cómo se hacen las cosas. Tales saberes aparecen bajo la forma de postulados pre-teóricos y de esquemas pragmáticos legitimadores de acciones profesionales concretas, de refranes y máximas que pautan el comportamiento propio y ajeno, de conceptos e ideas teóricas de mayor nivel de abstracción y formalización derivados de campos disciplinares específicos, etc."

Postulamos aquí que la formación profesional bajo un enfoque por competencias debe considerar a las TP: (a) como un insumo para llegar a constituirlas como TC, porque es un saber avanzado y situado, que ya ha demostrado en una comunidad profesional que es útil y por lo tanto, requiere ser considerado para lograr una mayor sistematización; (b) como un saber más cercano a la realidad profesional desde donde enseñar TC, es decir, que las TP representan un conocimiento de base sobre el cual se pueden enseñar TC, logrando así una mayor pertinencia; y (c) tal como lo propone Nolín (2008), como un saber que, aunque menos válido, confiable o creíble desde el mundo académico-científico, opera y resuelve problemas profesionales, por lo que merece ser difundido, enseñado y sometido a escrutinio.

Estas TP suelen estar presentes en la formación profesional tradicional, muchas veces socializadas por aquella parte del profesorado universitario que combina el ejercicio profesional con la docencia, dada la diversidad de tipos de universidades (en algunas de ellas este perfil de profesor puede llegar a ser mayoritario). No obstante su presencia en la formación, estas TP no se las suele diferenciar ni trabajar como un saber propiamente tal, es decir, el estudiante no conoce la ruta acerca de cómo la comunidad profesional llegó a elaborar dicha teoría. "La reflexión sentida en y sobre la práctica" (Pérez, 2010, p. 101), podría constituir una herramienta importante para unir la teoría científica con este saber más cercano a la práctica (Lampert, 2010).

El tratarlas como saber (analizable, discutible, conocible, transformable) ayudaría a su mejor integración con las TC y a la complejización o cambio a veces necesario, de estas TP, a la vez que podría resolver la dicotomía "teoría / práctica" o "teoría vs la realidad" que se incorpora rápidamente al pensamiento del profesional en formación. Una propuesta es que el currículum de formación incorpore en cada ciclo similares 
proporciones de módulos científicos y módulos práctico-profesionales, superando la tradición de reservar estos últimos para el final de la formación, en donde la discusión del conocimiento científico relativo a esa práctica disminuye. No favorece a la construcción de un pensamiento profesional de calidad, el que estudiantes estén aprendiendo ciencia básica sin poder relacionarla a escenarios práctico profesionales, ni que luego en el último año de formación, estén inmersos en escenarios práctico-profesionales lejos de la investigación relacionada a ellos.

Tampoco lo favorece si estas experiencias de aprendizaje situadas en contextos sociales no guían la integración de las TP con las TC, siendo incluso posible que esto contribuya más aún a las dicotomías señaladas. Por otra parte, el saber científico debe aprovechar el saber profesional, alejándose de la perspectiva de la posición de superioridad que lo caracteriza respecto al ejercicio profesional. Un ejemplo, en un estudio sobre teorías de profesionales (subjetivas) que trabajaban en escuelas de lenguaje en Chile, donde participó uno de los autores de este trabajo (Keyserlingk et al., 2013), se encontró que existía un consenso entre ellos respecto a identificar como causa de un trastorno, un aspecto que la investigación y el conocimiento científico excluye. Desde esto, surgen como preguntas: ¿es que han sido mal formados?, ¿por qué a pocos años de ejercicio profesional razonan de manera contraria a lo aprendido?. Sin embargo, otra forma de ver este tipo de resultados es preguntarse: ¿no será que la TC, generada la mayor parte de las veces en contextos culturales diferentes al nuestro, es la que no está siendo una buena explicación del fenómeno?

Las experiencias educativas centradas en la incorporación de los estudiantes en los contextos de práctica profesional y preprofesional, parecen ser la vía más valorada para un trabajo reflexivo y sistemático con las TP. Otros modelos formativos se han aproximado mediante un Aprendizaje Basado en Problemas (ABP) y Aprendizaje Servicio, contactando así al estudiante con la realidad social. Otras alternativas valiosas pueden ser los seminarios, la invitación de profesionales a dictar alguna charla en la universidad o a impartir asignaturas modularmente en conjunto con los(as) "científicos(as)", la conformación de núcleos de investigadores(as) con participación de profesionales en ejercicio y la difusión del ejercicio de su profesión mediante libros y artículos. Esto podría ser un primer paso para lograr la integración entre el saber profesional y el saber científico, siempre y cuando se incluya como parte del proceso formativo la reflexión sistemática sobre la práctica, desde el saber científico y también desde el conocimiento subjetivo.

\section{REFLEXIONES FINALES}

Los problemas que actualmente enfrenta nuestra sociedad son urgentes de resolver y frente a estos, los profesionales ocupan un rol cada vez más importante (Evetts, 2014). Así, en la disyuntiva contemporánea del impacto de las profesiones (Nolin, 2008), la pregunta de ¿para qué formar profesionales?, sugiere como respuesta, aunque no la única, la de ocupar un rol importante en la comprensión y solución de las dificultades que enfrentan las presentes y futuras generaciones, sobre todo las de tipo medio ambientales. Para lo anterior se requiere de una formación de calidad, que implique el desarrollo integral del futuro profesional (Bustamante et al., 2017; Mukhongo, 2015; Tobón, 2008) y contribuya a la construcción de una identidad profesional que vaya más allá de (a) la racionalidad técnica, productiva y de menor autonomía que el modelo mercantilista le atribuye a los profesionales; y por contraparte, (b) del sentido profesional que se centra en incrementar el valor de la profesión, más que en resolver los problemas de interés público (Evetts, 2014; Saks, 2016).

Parte de esta integralidad la compone aquella dimensión relacionada con los distintos tipos de saberes que inciden en la acción (Schön, 1984; Tobón, 2008), que son parte y dan forma a esta identidad profesional, sobre los cuales es necesario instalar procesos reflexivos durante la formación profesional. Se trata de una transdisciplinariedad para la formación profesional, siendo uno de sus imperativos la integración de estos saberes, para la comprensión y abordaje complejo de los problemas actuales (Morín, 2010). Epistemológicamente y desde una mirada psicológica, la construcción del conocimiento profesional implica la interrelación e interdependencia de los modelos de conocimiento explicativo que el profesional va desarrollando: se trata de que éste elabore una teoría compleja o de mayor complejidad, más articulada, con cadenas de relaciones, que oriente su ejercicio profesional. Esto puede ser un aspecto distintivo de las profesiones y que probablemente se relacione con la tan anhelada autonomía profesional sustentada en la reflexión (Schön, 1984), porque las teorías permiten describir y explicar los fenómenos y el propio comportamiento, además de justificar y orientar la acción.

Nuestra propuesta es que la formación profesional también debe considerar la construcción y reconstrucción de teorías por parte de los estudiantes (científicas, profesionales y subjetivas) y una adecuada interrelación entre éstas, para la formación integral e incluso, la construcción reflexiva, planificada, guiada o sistematizada de la identidad profesional. Porque las instituciones formativas principalmente han logrado sistematizar la enseñanza de aquel conocimiento que es científico-técnico, pero no se ha abordado con la 
suficiente preocupación al conocimiento que más influye en la identidad de los profesionales. Desde los procesos formativos formales y sobre todo desde un enfoque por competencias, se requiere un cambio de paradigma.

\section{Cambio de paradigma}

Implica poner en un mismo estatus a las TC, TP y TS, para el desarrollo de profesionales integrales. Es un reconocimiento de que el profesional actual necesita integrar nuevas formas de conocimiento (Nolin, 2008). Desde el enfoque de formación basado en competencias (Tobón, 2008), esto puede tener una importante función, porque los procesos formativos pueden orientarse no solo desde la estructura socioeconómica y la racionalidad técnica (Saks, 2016), sino que además desde los sentidos de los estudiantes, profesionales y beneficiarios de los servicios profesionales, representando un insumo desde donde se puede situar y orientar el proceso formativo. También desde los modelos explicativos que los profesionales elaboran desde su práctica profesional, porque son quienes conocen de primera fuente los problemas que les demandan soluciones. Junto a lo anterior, el cambio de paradigma supone concebir al aprendiz de alguna profesión, como un sujeto activo que puede relacionarse respectivamente con los distintos tipos de saberes (científico, profesional y cotidiano) desde una posición pasiva (en $3^{\underline{a}}$ persona), relacional (en $2^{\underline{a}}$ persona) y proactiva como constructor de teorías subjetivas (en 1ª persona) (Araya-Véliz et al., 2017).

Integrar los tres tipos de teorías (TS, TP y TC) requiere de un proceso que debe ser conducido con aquella intención. Desde diversas líneas de investigación acerca de la cognición humana (representaciones sociales de estudiantes de ciencias, teorías implícitas de estudiantes, teorías subjetivas de profesionales, cambio conceptual o creencias epistemológicas de estudiantes), se argumenta que este no es un proceso que se dé "naturalmente". Desde estas aproximaciones se ha observado que las personas recurren a diferentes tipos de conocimiento, según la demanda o escenario de actuación y que el conocimiento cotidiano muchas veces se impone sobre el científico, a la hora de explicar una situación concreta para la que existe una mejor explicación de naturaleza científica.

Un ejemplo reciente de lo anterior, realizado en nuestro contexto de educación superior: se observó que estudiantes de carreras de la salud recurrían a creencias epistemológicas sofisticadas al argumentar sobre un problema, sin embargo, al enfrentarse a preguntas de índole práctico-profesional basaban sus respuestas en creencias más ingenuas discordantes a las anteriores, más que eso, se observó que estudiantes de medicina más cercanos al ejercicio profesional, tenían creencias más objetivistas acerca del conocimiento en comparación a estudiantes de primer año (Lazcano, Villalón, Vera y Conget, 2017).

Así, el desafío de integración de los tipos de teorías supone de un proceso pedagógico intencionado. Desde una base epistemológica la integración supone acercar la primera persona con la tercera persona en espacios relacionales (segunda persona) (Araya-Véliz, et al., 2017) como la reflexión colectiva sistemática (Catalán-Ahumada y Castro, 2016). Desde una mirada pedagógica, supone que el docente asuma la posibilidad de coexistencia y de integración de los tipos de conocimiento y no la ilusión de la sustitución del conocimiento cotidiano por el científico o profesional. La anterior visión requiere que el conocimiento cotidiano sea explicitado no para mostrar su falta de validez ni para considerarlo un "obstáculo epistemológico" como señalan Hilger et al. (2017 p. 1303-8.), sino para que el aprendiz sea consciente de sus TS y de las limitaciones, continuidades y discontinuidades en relación a los otros tipos de conocimiento que aprende durante su formación (TC y TP).

Desde el estudio de las teorías subjetivas, por ejemplo, de profesionales de la educación, lo anterior se ha propuesto a través de la reflexión de las TC, en relación a las TS y la práctica, como medio para la transformación del conocimiento (Uljens, 1997). Transformación entendida en el presente trabajo no como la sustitución de un tipo de conocimiento por otro, cuestión que requiere de cambios conceptuales radicales difíciles de lograr, sino la integración de ellos con miras al desarrollo de un pensamiento complejo como competencia a lograr, en donde el estudiante y futuro profesional haga un "uso" no ingenuo (consciente, explícito y reflexivo) de sus teorías personales diferenciándolas o integrándolas, cuando corresponda según el contexto de aplicación profesional. La tabla 1 permite comparar cada teoría según algunas de sus características.

\section{Orientar el aprendizaje hacia un cambio subjetivo}

Si bien desde el enfoque de formación basado en competencias se propone como meta promover aprendizajes que, a fin de cuentas, suponen cambios a nivel cognitivo, afectivo y volitivo; la subjetividad del estudiante para promover este tipo de aprendizaje complejo, no ha sido considerada con la suficiente elaboración y sistematización. 
Se trata de incorporar estrategias pedagógicas orientadas a reconstruir e incorporar como parte de la enseñanza, las TS de los futuros profesionales o los que se encuentran en formación continua. La evidencia científica muestra que este conocimiento subjetivo tiene una implicancia importante en la disposición o actitud ante el aprendizaje y la forma en que se le otorga sentido a los problemas relacionados con el ejercicio profesional, regulando sus prácticas en base a éstas sobre todo cuando desde la racionalidad técnica no encuentran respuesta (Šteh et al., 2014). Esta subjetividad permite otorgar un sentido a la propia vida y la que transcurre más allá de esta, por lo que constituye un ámbito importante de la identidad personal y profesional. Al respecto, hay propuestas que surgen desde las teorías de cambio de TS (Castro et al., 2015; Catalán 2016) y de cambio representacional (Pozo, 2002), que básicamente proponen: (a) hacer explícitas estas teorías de los estudiantes, (b) promover un reconocimiento y comprensión sobre cómo éstas orientan su forma de comportarse, sobre todo cómo se asume el cambio o aprendizaje; (c) reflexionar sobre la funcionalidad de esta forma de pensar; (d) enriquecerlas mediantes nuevas fuentes de conocimiento.

\section{Identificar, reconstruir y considerar en la enseñanza a las teorías profesionales}

Es preciso considerar las TP como modelos explicativos muy asociados al desempeño profesional situado, es decir, como un conocimiento que orienta su acción en el ejercicio de su profesión y que en su comunidad profesional ha demostrado utilidad (Andreozzi, 2011; Campillo et al., 2012; Schön, 1984; Sosa y Ribeiro, 2015). Esto significa que la formación profesional requiere además el aprendizaje de estas teorías, poniendo como eje de su enseñanza la reflexión. La teorización sobre las prácticas, parece ser “... la estrategia más adecuada para la formación de sus competencias profesionales, que no son simplemente contenidos que se recitan, sino plataformas, herramientas intelectuales, sistemas de comprensión, diagnóstico, planificación, actuación y valoración..." (Pérez y Pérez, 2013, p. 79) de este saber más cercano a la práctica.

La socialización profesional ha constituido una de las principales herramientas pedagógicas para aprender este tipo de conocimiento (Andreozzi, 2011), por ejemplo, mediante prácticas tempranas o profesionales. Sin embargo, el desafío queda puesto en cómo reconstruir este tipo de teorías a veces subjetivas y otras veces consensuadas u objetivadas socialmente, para sistematizar su enseñanza. Por lo pronto, una mínima condición necesaria es que la investigación de las profesiones incluya como objeto de estudio a las TP y su enseñanza-aprendizaje. Sin embargo, también es necesario discutir epistemológicamente las características de este conocimiento y sobre nuevos métodos de sistematización, reconstrucción y enseñanza del mismo.

\section{El desarrollo del pensamiento superior}

En este trabajo se ha destacado la necesidad de integración de estos tres saberes para lograr en el profesional un desempeño idóneo. Para esto, se requiere el desarrollo del pensamiento de orden superior, que se revela en la actividad instrumental y en la interacción social; en espacios de mediación semiótica que permiten acceder a formas de control consciente y voluntario del comportamiento (Vygotsky, 1978). En forma contemporánea, esta forma de actividad conjunta puede entenderse también como una práctica reflexiva y autorregulada. Ello puede surgir en las comunidades de práctica, tanto en la formación, como en la demostración de competencias profesionales.

Van Velzen (2017) plantea que el conocimiento metacognitivo y la solución de problemas caen en la categoría de pensamiento de orden superior y permiten desarrollar competencias para enfrentar la solución de problemas complejos en forma no rutinaria. Este pensamiento incluye en forma sistemática y autorreflexiva, el uso expandido y combinado de competencias cognitivas tales como análisis, evaluación y síntesis, en base a las cuales el profesional puede llegar a integrar las TC, TP y TS. Por su parte, Sanz de Acedo (2010), plantea competencias cognitivas en la formación y actuación profesional. Entre ellas se destacan las relacionadas con el pensamiento comprensivo, el pensamiento crítico, el pensamiento creativo y las competencias complejas que incluyen la toma de decisiones y solución de problemas. Todas las anteriores requieren de la metacognición, la autorregulación y la transferencia como recursos necesarios para su desarrollo continuo. Así, en el proceso de desarrollo de las competencias profesionales, la transición a un nivel de experto requiere de una activa reflexión sobre aspectos críticos de la práctica profesional, para de esta forma, superar la autocomplacencia y acceder a una competencia reflexiva y consciente (Hallam, 2009). Ello demanda negociar múltiples transiciones entre contextos diversos de tipo profesional-disciplinario, superando el pensamiento intuitivo (Vosniadou, 2008), y desarrollar una autorregulación consciente, lo que lleva al profesional a continuas restructuraciones de las TC, TP y TS que sustentan su acción. Más aun, la falta de reflexión metacognitiva y autorregulada puede ser un obstáculo para alcanzar un nivel experto en las competencias profesionales, bloqueando las creencias de autoeficacia y la apertura a un autoaprendizaje (Hallam, 2009) que permita el enriquecimiento de los modelos explicativos para proporcionar respuestas oportunas y de impacto social positivo. 
Tabla 1: Comparación entre teorías científicas, profesionales y subjetivas

\begin{tabular}{|c|c|c|c|c|c|}
\hline Teorías & Génesis & $\begin{array}{c}\text { Nivel de } \\
\text { Sistematización }\end{array}$ & $\begin{array}{c}\text { Contexto en que } \\
\text { Aplican }\end{array}$ & $\begin{array}{c}\text { Aplicabilidad } \\
\text { en el } \\
\text { Ejercicio } \\
\text { Profesional }\end{array}$ & $\begin{array}{c}\text { Posición } \\
\text { Perceptual del } \\
\text { Aprendiz y de } \\
\text { Acceso a este } \\
\text { Tipo de Teorías }\end{array}$ \\
\hline $\begin{array}{l}\text { Teorías } \\
\text { científicas }\end{array}$ & $\begin{array}{l}\text { Elaboradas por } \\
\text { científicos en base a } \\
\text { métodos científicos } \\
\text { Saber que se } \\
\text { objetiviza, sistematiza y } \\
\text { somete a juicio en la } \\
\text { comunidad científica }\end{array}$ & $\begin{array}{l}\text { Alto } \\
\text { Conocimiento } \\
\text { "objetivo" }\end{array}$ & $\begin{array}{l}\text { 1. Campo científico- } \\
\text { académico } \\
\text { 2. Campo } \\
\text { profesional } \\
\text { 3. Menos en la vida } \\
\text { cotidiana }\end{array}$ & $\begin{array}{l}\text { Aplicables a } \\
\text { algunos } \\
\text { problemas } \\
\text { profesionales }\end{array}$ & $\begin{array}{l}3^{\text {a }} \text { persona } \\
\text { Observador } \\
\text { lejano y } \\
\text { abstracto } \\
\text { (Araya-Véliz et } \\
\text { al., 2017) }\end{array}$ \\
\hline $\begin{array}{l}\text { Teorías } \\
\text { profesionales }\end{array}$ & $\begin{array}{l}\text { Elaboradas por } \\
\text { profesionales desde la } \\
\text { práctica o ejercicio } \\
\text { profesional, reflexión } \\
\text { sobre la práctica y } \\
\text { socialización } \\
\text { profesional. Saber que } \\
\text { no es objetivo ni } \\
\text { subjetivo, que se valida } \\
\text { en una comunidad de } \\
\text { profesionales a partir } \\
\text { de su aplicabilidad y } \\
\text { utilidad profesional }\end{array}$ & $\begin{array}{l}\text { Menos } \\
\text { sistemático } \\
\text { Saber válido, a } \\
\text { veces subjetivo } \\
\text { y otras veces } \\
\text { consensuado u } \\
\text { objetivado } \\
\text { socialmente e } \\
\text { históricamente }\end{array}$ & $\begin{array}{l}\text { Principalmente en el } \\
\text { ejercicio profesional, } \\
\text { cuando la } \\
\text { racionalidad técnica } \\
\text { no tiene respuestas } \\
\text { frente a los } \\
\text { problemas que } \\
\text { enfrentan }\end{array}$ & $\begin{array}{l}\text { Aplicables a } \\
\text { la mayoría de } \\
\text { los } \\
\text { problemas } \\
\text { profesionales }\end{array}$ & $\begin{array}{l}2^{\text {a }} \text { persona } \\
\text { Constructor de } \\
\text { saber en la } \\
\text { experiencia y en } \\
\text { relación con } \\
\text { otros }\end{array}$ \\
\hline $\begin{array}{l}\text { Teorías } \\
\text { subjetivas }\end{array}$ & $\begin{array}{l}\text { Elaboradas por todas } \\
\text { las personas en y para } \\
\text { las experiencias de la } \\
\text { vida cotidiana y la } \\
\text { interacción social } \\
\text { (Catalán, 2016) } \\
\text { Saber subjetivo que } \\
\text { puede ser implícito o } \\
\text { explícito }\end{array}$ & $\begin{array}{l}\text { Bajo } \\
\text { Saber válido, } \\
\text { intuitivo y } \\
\text { subjetivo }\end{array}$ & $\begin{array}{l}\text { 1. Principalmente en } \\
\text { la vida cotidiana } \\
\text { 2. En el ejercicio } \\
\text { profesional, si las } \\
\text { teorías científicas y } \\
\text { profesionales no les } \\
\text { permiten resolver } \\
\text { problemas }\end{array}$ & $\begin{array}{l}\text { Aplicables a } \\
\text { los } \\
\text { problemas } \\
\text { profesionales } \\
\text { que no tienen } \\
\text { respuesta } \\
\text { desde las } \\
\text { teorías } \\
\text { científicas y } \\
\text { profesionales }\end{array}$ & $\begin{array}{l}\text { 1a persona } \\
\text { Elaborador de } \\
\text { sus propias } \\
\text { teorías } \\
\text { subjetivas } \\
\text { (Catalán, 2016) }\end{array}$ \\
\hline
\end{tabular}

Por otra parte, desde una perspectiva epistemológica, este pensamiento de orden superior se entiende como una forma de reflexión que se centra en los modos de producción de conocimientos (TC, TP y TS), que pone a prueba las certidumbres racionales, teóricas, metodológicas, así como las mismas interpretaciones socialmente aceptadas sobre la realidad social. Siguiendo a Bachelard (2000), se plantea la necesidad de formar profesionales de las ciencias sociales para la vigilancia epistemológica acerca del conocimiento y sus implicancias disciplinarias y profesionales. Esta vigilancia se hace más necesaria cuando no existen procedimientos estables y útiles frente a todas las situaciones en el acercamiento a la realidad social (Farías, 2009), en este caso, las situaciones que implican contenidos curriculares y prácticas pedagógicas.

Para finalizar, es preciso señalar que la formación profesional de calidad hoy más que nunca es parte de la respuesta a los complejos problemas socio ambientales de la humanidad. La formación integral no se agota con el desarrollo de un conocimiento profesional más holista, aunque sí da algunas luces acerca de la implicancia que tiene esto en la construcción de una identidad profesional y en el ejercicio de la profesión y, por lo tanto de cómo esto se podría considerar desde un enfoque formativo basado en competencias.

\section{CONCLUSIONES}

(i) En este trabajo se ha propuesto que el desempeño profesional idóneo requiere de una formación profesional que incluya tres saberes: TC, TP y TS; (ii) La formación universitaria ha puesto un mayor énfasis en la enseñanza del saber científico técnico, en donde las TC ocupan un rol protagónico. Sin embargo, esta 
forma de enseñanza no ha logrado una adecuada relación entre la teoría y la práctica; (iii) Las TP y las TS representan aquellos saberes más situados y cercanos a la experiencia y práctica profesional, por lo que se torna imperativo incluirlos sistemáticamente en los procesos formativos; y (iv) La integración de estos tres saberes puede conllevar al desarrollo de una teoría razonada, integrada, compleja y más funcional en los profesionales en formación, que les permita un abordaje más efectivo de los complejos problemas sociales y ambientales que nuestra sociedad enfrenta.

\section{REFERENCIAS}

Andreozzi, M., Las Prácticas Profesionales de Formación como Experiencias de Pasaje y Tránsito Identitario, Archivos de Ciencias de la Educación, ISSN: 2346-8866, 5(5), 99-11 (2011)

Araya-Véliz, C., R. Arístegui y P. Fossa, Pasos Hacia una Enacción Relacional. Aporte, Ambigüedades y Limitaciones del Concepto Embodied Mind en Francisco Varela: un Análisis Metateórico. Mindfulness \& Compassion, 2(1), 41-46 (2017)

Bachelard, F., La Formación del Espíritu Científico, Contribución a un Psicoanálisis del Conocimiento Objetivo, 23ª Ed., Siglo Veintiuno, México (2000)

Bustamante, M., M. Lapo, C. Oyarzún y R. Campos, Análisis de la Percepción del Docente en Tres Universidades Chilenas tras la Implementación del Currículum Basado en Competencias, doi:10.4067/S0718-50062017000400009, Formación Universitaria, 10(4), 97-110 (2017)

Campillo, M., J. Sáez y F. Del Cerro, El Estudio de la Práctica y la Formación de los Profesionales, Revista de Educación a Distancia, (6), 1-23 (2012)

Castro, P., M. Krause y S. Frisancho, Teoría del Cambio Subjetivo: Aportes desde un Estudio Cualitativo con Profesores, doi:10.15446/rcp.v24n2.44453, Revista Colombiana de Psicología, 24(2), 363-379 (2015)

Catalán, J., Hacia la Formulación de una Teoría General de las Teorías Subjetivas, doi: 10.5027/psicoperspectivasvol15- issue1-fulltext-739, Psicoperspectivas. Individuo y Sociedad, 15(1), 53-65 (2016)

Catalán-Ahumada, J. y Castro, P.J. Reflexión Colectiva Sistemática: un Estudio Orientado al Desarrollo Profesional Docente, doi: 10.1590/2175-353920150201949, Psicol. Esc. Educ, 20(1), 157-167 (2016)

Donoso, S. y O. Corvalán, Formación Técnica y Aseguramiento de la Calidad: Enfoque de Desarrollo de Competencias, Cadernos de Pequisa, 42(1), 612-639 (2012)

Evetts J., The Concept of Professionalism: Professional Work, Professional Practice and Learning. En S. Billett, C. Harteis y H. Gruber (Eds.). International Handbook of Research in Professional and Practice-based Learning, Springer, Dordrecht, Países Bajos, 29-56 (2014)

Farías, F., La Epistemología de las Ciencias Sociales en la Formación por Competencias del Pregrado, https://dx.doi.org/10.4067/S0717-554X2009000100004, Cinta de Moebio, (34), 58-66 (2009)

Flick, U., An Introduction to Qualitative Research, 5프. Ed., Sage, Londres, Inglaterra (2014)

Follari, R., Teoría Científica y Práctica Profesional: Relaciones no Lineales e Imprescindibles, Revista Pilquen, 13(2), 3947 (2016)

Frade, L., Desarrollo de Competencias en Educación: desde Preescolar hasta el Bachillerato, Inteligencia Educativa, DF, México (2009)

Gallegos, M., M. Berra, E. Benito y W. López, Las Nuevas Dinámicas del Conocimiento Científico y su Impacto en la Psicología Latinoamericana, doi:10.5027/psicoperspectivas-vol13-issue3-fulltext-377, Psicoperspectivas. Individuo y Sociedad, 13(3), 106-117 (2014)

Gergen, K., Realidades y Relaciones, Aproximaciones a la Construcción Social, Paidós, Barcelona, España (1996)

Hallam, S., Transitions and the Developmentof Expertise, Psychology Teaching Review, 16(2), 3-32 (2009)

Hilger, T.R., M.S. Stipcich y M.A. Moreira, Representações Sociais sobre Física Quântica entre Estudantes de Graduação Brasileiros e Argentines, Latin-American Journal of Physics Education, 11(1), 1303-1 1303-9 (2017)

Hill, J. y P. Houghton, A Reflection on Competency-based Education: Comments from Europe, JME, 25(2), 146-166 (2001)

Juliá, Ma .T., Competencias Generales de la Formación Universitaria: Aportes a la Calidad con Equidad. En G. Carrillo (Ed.), I Encuentro Internacional Universitario. El Currículum por Competencias en la Educación Superior. Ponencias y Debates. Pontificia Universidad Católica del Perú, Lima, Perú, 15-38 (2015)

Keyserlingk, L. von, P. J. Castro y J. Carrasco, Teorías Subjetivas de Profesionales de Escuelas de Lenguaje en Chile sobre el Trastorno Específico del Lenguaje, doi: 10.1590/S1516-18462013005000039, Cefac, 15(4), 873-883 (2013)

Kindermann, K. y U. Riegel, Subjektive Theorien von Lehrpersonen. Variationen und Methodische Modifikationen eines Forschungsprogramms, Forum Qualitative Sozialforschung, ISSN: 1438-5627, 17(2), Art. 1 (2016)

Lago, D. y R. Ospina, Diseño Curricular Basado en Competencias: el Caso del Doctorado en Ciencias de la Educación de la Universidad de Cartagena. En G. Carrillo (Ed.), I Encuentro Internacional Universitario. El Currículum por Competencias en la Educación Superior. Ponencias y Debates, Pontificia Universidad Católica del Perú, Lima, Perú (2015) 
Lampert, M., Learning Teaching in, from, and for Practice: What Do We Mean? doi:10.1177/0022487109347321, Journal of Teacher Education, 61(1-2), 21-34 (2010)

Lazcano, X., F. Villalón, S. Vera y P. Conget, La Creencia Epistemológica Constructivista sobre el Conocimiento Científico Varía en Función del Año de Formación en los Estudiantes de Medicina pero no en los Estudiantes de otras Carreras de la Salud, doi: 10.4067/s0034-98872017000901184, Revista Médica de Chile, 145(9), 1184-1192 (2017)

Morín, E., Complejidad Restringida, Complejidad General, Revista Estudios, 8(93), 81-135 (2010)

Mukhongo, L., Competence-based Training in Higher Education Institutions, IJSRSET, 1(5), 99-105 (2015)

Nolin, J., In Search of a New Theory of Professions, Science for the Professions, University of Borås, 4, 5-53 (2008)

Pérez, A., Aprender a Enseñar en la Práctica: Procesos de Innovación y Prácticas de Formación en la Educación Secundaria, Graó, Barcelona, España (2010)

Pérez, A. y L. Pérez, Competencias Docentes en la Era Digital, La Formación del Pensamiento Práctico, Temas de Educación, 19(1), 67-84 (2013)

Pozo, J., La Adquisición de Conocimiento Científico como un Proceso de Cambio Representacional, Investigações em Ensino de Ciências, 7(3), 245-270 (2002)

Red Psicología CUECH Proyecto MECESUP ULS 0601, Competencias del Perfil de Egreso de Psicólogo. En Mª.T. Juliá (Ed.), Competencias del Psicólogo en Chile, Propuestas desde las Universidades Estatales, 166 - 188, Editorial Universidad de La Serena, La Serena, Chile (2013)

Rodrigo, Mํ...J., El Hombre de la Calle, el Científico y el Alumno, Novedades Educativas, 76, 59-66 (1997)

Saks, M., A Review of Theories of Professions, Organizations and Society: The Case for Neo - Weberianism, Neo Institutionalism and Eclecticism, doi: 10.1093/jpo/jow005, JPO, 3(2), 170-187 (2016)

Sanz de Acedo Ma‥L., Competencias Cognitivas en Educación Superior, Narcea Ediciones, Madrid, España (2010)

Schomburg, H. y U.Teichler, Higher Education and Graduate Employment in Europe Results from Graduates Surveys from Twelve Countries, doi: 10.1007/978-1-4020-5154-8, Series: Higher Education Dynamics, 15, Springer, Dordrecht, Paises Bajos (2006)

Schön, D., The Reflective Practitioner: How Professionals Think In Action, Basic Books, New York, USA (1984)

Sosa, L. y C. Ribeiro, Conocimiento Profesional como Característica Distintiva de Profesionalización Docente en la Formación de Profesores, Revista Iberoamericana de Producción Académica y Gestión Educativa, 2, 1-19 (2015)

Šteh, B., J. Kalin y J. Mažgon, The Role and Responsibility of Teachers and Students in University Studies: a Comparative Analysis of the Views Expressed by Pedagogy Students, doi: 10.2298/ZIPI1401050S, Зборник Института за педагошка Uстраживања, 46, 50-68 (2014)

Tamir P., Professional and Personal Knowledge of Teachers and Teachers Educators, Teacher and Teaching Education, 7(3), 263-268 (1991)

Tobón, S., Formación Basada en Competencias, Pensamiento Complejo, Diseño Curricular, Editorial Ecoes, Colombia (2001)

Tobón, S., La formación Basada en Competencias en la Educación Superior: El Enfoque Complejo, Grupo CIFE, Guadalajara (2008)

Tobón, S., J. Pimienta y J. García, Secuencias Didácticas: Aprendizaje y Evaluación de Competencias, Pearson Educación, México (2010)

Uljens, M., School Didactics and Learning: a School Didactic Model Framing an Analysis of Pedagogical Implications of Learning Theory, Psychology Press, United Kingdom (1997)

van Velzen, J., Metacognitive Knowledge: Development, Application, and Improvement, Information. Age Publ. Inc., EEUU (2017)

Vigotsky. L., Psicología de los Procesos Psicológicos Superiores, Paidós, Madrid (1978)

Vosniadou, S., Conceptual Change Research: an Introduction. En S. Vosniadou (Ed.). International Handbook of Research on Conceptual Change, Routledge, London, xiii-xxviii (2008) 
